\title{
DISSECÇÃO ESPONTÂNEA DE ARTÉRIA CARÓTIDA INTERNA COM PARALISIA DE NERVOS CRANIANOS INFERIORES
}

Ao Editor - Gostaria de cumprimentar os autores do trabalho "Dissecção espontânea de artéria carótida interna com paralisia de nervos cranianos inferiores"1 pelo caráter pedagógico do mesmo ressaltando forma de apresentação não comum das dissecções carotídeas, no entanto acredito ser pertinente algumas considerações no sentido de estimular a discussão. Atualmente, a opção terapêutica para a doença cerebrovascular isquêmica sintomática aterotrombótica ou tromboembólica, seguindo o protocolo específico, se assim o ambiente médico permitir, é o uso de trombolítico (rt - PA) ${ }^{2}$ aplicando-se inclusive nos quadros de doença cerebrovascular isquêmica aguda relacionada a dissecção espontânea de artéria carótida interna (DEACl) ${ }^{3,4}$. Uma vez fechada a janela terapêutica na fase hiperaguda, o que fazer? Qual a história natural da $D E A C l$ ? Há justificativa para o uso de anticoagulante ou antiagregante plaquetário a longo prazo? Kremer et al. ${ }^{5}$ concluíram em seu estudo que a $\mathrm{DEACl}$ tem um prognóstico a longo prazo benigno com reduzido risco ( 0.3 a $0.6 \%$ ao ano) de acidente cerebrovascular isquêmico carotídido ipsilateral. Por outro lado, não há recomendações evidentes para utilizar anticoagulante ou antiagregantes plaquetários a não ser indícios da participação predominante do mecanismo troboembólico sobre o mecanismo hemodinâmico baseado em estudo com Doppler Trascraneano que relataram a presença de microembolizações nas DEACl em $59 \%$ dos casos avaliados ${ }^{6,7}$. O prognóstico da DEACI na verdade depende do padrão evolutivo das alterações vasculares: lesões estenóticas tendem a sofrer um processo de remodelação espontânea em torno de $70 \%$ dos casos muitas delas sem seqüelas visíveis, enquanto as oclusões nas DEA$\mathrm{Cl}$ tendem a recanalizar em $90 \%$ dos casos $^{8}$. Tem-se demonstrado nas DEACl que em torno de $50 \%$ dos casos, apresentam alterações no colágeno e na elastína de biópsias de pele denotando uma forte associação com as desordens do tecido conectivo ${ }^{9}$. Assim uma arteriopatia dada por um desarranjo conectivo em uma base de predisposição genética tem sido sugerido como patogênese para a formação de um coagulo entre as camadas da artéria (um verdadeiro acidente vascular hemorrágico) com ou sem a formação adicional de trombo intraluminal seja pela estase da estenose ou, muito mais, pela ruptura da íntima com trombogênese secundária.

A DEACl parece ser um processo de natureza muito dinâmica tendo como base dois aspectos: uma lesão expansiva hemorrágica intra arterial com ou sem um trombo intravascular adicional, devendo ser avaliada preferencialmente na fase hiperaguda por meio de RNM com supressão de gordura, angio-RNM e arteriografia digital complementar com o objetivo de planejar qual a melhor opção terapêutica: terapia trombolítica endo- venosa e ou intra arterial combinada ou não a angioplastia e stent auto expanssivel $^{10}$. Uma vez fechada a janela terapêutica da fase hiperaguda, descartando doenças hereditária do tecido conectivo e displasia fibromuscular e considerando os indícios de uma boa história natural evolutiva, penso que a monitorização radiológica da arteria carótida interna dissecada e suas possíveis repercussões hemodinâmicas e tromboembólicas monitoradas com Doppler Carotídeo-Transcraniano nos orientarão na mais adequada conduta a tomar em relação às terapias antitrombóticas ${ }^{7}$ e de remodelação vascular por meio de embolização dos pseudo aneurismas e ou o uso de angioplastia com stent carotídeo se ainda não tiver sido empregada. No contexto evolutivo do processo, talvez nada seja necessário, caso a doença siga o seu curso de reperfusão e remodelação da parede do vaso de forma espontânea, denotando reduzido risco de eventos isquêmicos secundários.

Para finalizar, gostaria de levantar a questão do uso de terapia antiagregante plaquetária com clopidogrel e AAS na manutenção a longo prazo do paciente, no caso descrito com DEACI. Estudos como CAPRIE $^{11}$, CURE $^{12}$, CREDO $^{13}$, MATH $^{14}$ e CHARISMA $^{15}$ indicam que o clopidogrel é somente algo "mais efetivo" que o AAS na prevenção secundária de eventos vasculares, estatisticamente evidente no subgrupo de pacientes com doença vascular periférica e com angina instável. Terapia combinada com clopidogrel e AAS é empregada como padrão nos procedimentos de angioplastia e stent carotídeo, coronariano e na angina instável como orientam os resultados obtidos em estudos como CURE, CREDO e CARESS ${ }^{16}$. Não há evidências convincentes de que clopidogrel ou AAS mais clopidogrel em comparação à AAS isoladamen- 
te melhore verdadeiramente a evolução de pacientes com doença cerebrovascular isquêmica a não ser incrementar em muito os custos do tratamento assim como o risco de complicações hemorrágicas de maior gravidade.

\section{REFERÊNCIAS}

1. Vieira VLF, Pereira DC, Ribeiro VT, Leite ABC, Emerique I. Dissecção espontânea de artéria carótida interna com paralisia de nervos cranianos inferiores. Arq Neuropsiquiatr 2006; 64:1047-1049.

2. Adams H, Adams R, Del Zoppo G, Goldstein LB. Guidelines for the early manegement of patients with ischemic stroke: 2005 guidelines update a scientific statement from the Stroke Council of the American Heart Association. Stroke 2005; 36:916-923.

3. Georgiadis D, Baumgartner RW. Thrombolysis in cervical artery dissection. Front Neurol Neurosci 2005;20:140-146.

4. Georgiadis D, Lancczik O, Schwab S, et al.. IV trombolysis in patients with acute stroke due to spontaneous carotid dissection. Neurology 2005;64:1612-1614.

5. Kremer C, Mosso M, Georgiadis D, et al.Carotid dissection with permanent and transient occlusion or severe stenosis. Neurology 2003;60: 271-275.

6. Wouter I Schievink. Spontaneous dissection of the carotid and vertebral arteries. N Engl J Med 2002;334:898-906.

7. Srinivasan J, Newell DW, Sturzenegger M, Mayberg MR, Wiinn HR. Trancranial Doppler in the evaluation of internal carotid artery dissection. Stroke 1996;27:1226-1230.

8. Touze E, Gauvrit JY, Meder JF, Mas JL. Prognosis of cervical artery dissection. Front Neurol Neurosci 2005;20:129-130.

9. Brandt T, Orberk E, Webwe R, et al. Pathogenesis of cervical artery dissections. Association with connective tissue abnormalities. Neurology 2001;57:24-30.

10. Dabitz R, Triebe S, Leppmeier U, Ochs G, Vorwerk D. Percutaneous recanalization of acute internal carotid artery occlusion in patients with severe stroke. Cardiovasc Intervent Radiol 2007;30:34-41.

11. CAPRIE steerig committee.A randomized blinded trial of clopidogrel versus aspirin in pacients at risk of ischemic events. Lancet 1996;348:1329-1339.

12. Yusuf S, Zhao F, Mehta SR, Chrolavicius S,Tognoni G, Fox KK; Clopidogrel in unstable angina to prevent recurrent events: trial investigators. Effects of clopidogrel in addition to aspirin in patient with acute coronary syndromes without ST segment elevation. N Engl J Med. 2001;345:494-502.

13. Steinhubl SR, Berger PB, Mann JT; for the CREDO investigators. Early and sustained dual oral antiplatelet therapy following percutaneous coronary intervention: a randomized controlled trial. JAMA 2002;288:2411-2420.

14. Diener HC, Bogousslavsky J, Brass LM, et al. and MATCH investigators. Aspirin and clopidogrel compared with clopidogrel alone after recent ischemic stroke or transient ischemic attack in high risk patients (MATCH): a randomimised, double blind, placebo controlled trial. Lancet 2004;364:331-337.

15. CHARISMA Investigators. Clopidogrel and aspirin versus aspirin alone for the prevention of atherotrombotic events. N Engl J Med 2006;345:1706-1717.

16. Markus HS, Droste DW, Kaps M, Larrue KR, Sieble M, Ringelstein EB. Dual antiplatelet therapy with clopidogrel and aspirin in symptomatic carotid stenosis evaluated using Doppler embolic signal detection: clopidogrel and aspirin for reduction of emboli in symptomatic carotid stenosis (CARESS) trial. Circulation 2005;111:2233-2240.

Heraldo Macuco Capella Neurologia Hospital Regional Dr Homero de Miranda Gomes São José SC - Brasil
Resposta da Autora - Gostaria de cumprimentar o colega pela excelência dos comentários.

Como já citado, a finalidade do artigo foi mostrar as bases anatômicas da dissecção espontânea da artéria carótida interna com paralisia dos últimos nervos cranianos. Por se tratar de um evento raro e de difícil diagnóstico, existe uma certa dificuldade, na grande maioria dos casos, em se fechar a janela terapêutica ainda na fase hiperaguda, o que acaba por dificultar o uso freqüente da terapia trombolítica. Apesar de não existirem evidências científicas, a abordagem terapêuticas desses pacientes ainda é feita baseada em anticoagulação e antiagregação plaquetária.

No que diz respeito aos aspectos fisiopatológicos, realmente é sabido da existência de alterações no colágeno e na elastina de biópsias de pele, mediante análise com microscopia eletrônica em paciente portadores sintomáticos e assintomáticos de Síndrome de Marfan e Síndrome de Ehlers Danlos. No entanto, na atual conjuntura nacional, o custo-benefício dessa abordagem propedêutica não é justificável, além de não alterar o curso da doença.

Mesmo sendo um exame pouco invasivo, o Doppler transcraniano não apresenta sensibilidade e especificidade suficientes que justifique a sua utilização para acompanhamento evolutivo do caso. 0 exame padrão-ouro, tanto para diagnóstico quanto para seguimento, ainda é a angiografia digital cerebral; mas com o passar dos anos, a angiorressonância cervical e craniana vem adquirindo sensibilidade e especificidade que justifique sua inclusão no arsenal de exames utilizados na investigação das dissecções arteriais cervicais.

\section{REFERÊNCIAS}

1. Vieira VLF, Pereira DC, Ribeiro VT, Leite ABC, Emerique I. Dissecção espontânea de artéria carótida interna com paralisia de nervos cranianos inferiores. Arq Neuropsiquiatr 2006; 64:1047-1049

2. Schievink WI. Spontaneous dissection of the carotid and vertebral arteries. N Eng Med J 2999;344 : 898

3. Arnold M, et al. Carotid and vertebral artery dissection. Pract Neurol 2005;5:100

4. Zweifler RM, et al. Arterial dissection. In Mohr JP, et al. Stroke New York: Churchill Livingstone, 2004

5. Lucas et al. Stroke patterns of internal carotid artery dissection in 40 patients. Stroke 1998;29:2648

6. Brandt $\mathrm{T}$, et al. Pathogenesis of cervical arterial dissection. Neurology 2001;57:24.

7. Donnan GA, et al. Extracranial arterial dissection. Stroke 2005;26 2043. 